\title{
A Patient of Crescentic Glomerulonephritis with Positive Anti Myeloperoxidase Antibodies and Immunoglobulin a Deposits on Immunofluorescence: The Conundrum Continues
}

\author{
Mayank Chawla ${ }^{1 *}$, Tan Puay Hoon ${ }^{2}$ and Shashidhar Baikunje ${ }^{1}$ \\ ${ }^{1}$ Department of General Medicine, Sengkang General Hospital, Singapore \\ ${ }^{2}$ Department of Pathology, Singapore General Hospital, Singapore
}

\begin{abstract}
Immunoglobulin (Ig)A nephropathy is the commonest type of primary glomerulonephritis (GN) in the world, with an estimated incidence of 2.5/100000 adults/year. It can co-exist with systemic conditions and is a common cause of crescentic GN. Antineutrophilic cytoplasmic antibody (ANCA) associated vasculitis can present as necrotizing, crescentic GN with pauci immune features on immunofluorescence on renal biopsy. There have been previous case reports of the presence of ANCA and IgA nephropathy in the same patient. Such patients more often have systemic signs and tend to have worse kidney function at presentation as compared to ANCA negative IgA nephropathy patients. However, they tend to show better response to immunosuppressive medication. We report a case of a young Asian lady who presented with acute kidney injury secondary to crescentic GN. She was found to have positive serum anti myeloperoxidase antibodies and also IgA deposits in the mesangium on renal biopsy. She was managed with steroids and cyclophosphamide, but her renal function did not improve after 6 months of induction therapy. This case reiterates the conundrum faced by the clinician in the interpretation of a combination of positive anti MPO antibodies with mesangial hypercellularity, positive mesangial staining for IgA and presence of crescentic nephritis. It also describes the variation in response to therapy in such cases.
\end{abstract}

Keywords

Acute kidney injury, Crescentic glomerulonephritis, Myeloperoxidase, Cyclophosphamide, IgA

\section{Introduction}

Immunoglobulin (Ig)A nephropathy is the commonest type of primary glomerulonephritis (GN) in the world, with an estimated incidence of 2.5/100000 adults/year [1]. It can co-exist with other systemic conditions and has been reported in a patient with Ig G- kappa multiple myeloma [2]. It is also a cause of crescentic GN. Histologically, IgA nephropathy is characterized by variable combinations of mesangial hypercellularity, endocapillary proliferation, segmental sclerosis, and tubulointerstitial changes, with or without crescents and significant mesangial staining with IgA, with or without IgM and C3 on immunofluorescence (IF).

Antineutrophilic cytoplasmic antibody (ANCA) associated vasculitis can present as necrotizing, crescentic $G N$ with pauci immune features on IF on the renal biopsy. The ANCAs can target either myeloperoxidase (MPO) or proteinase-3 (PR3).

There have been previous case reports of the presence of ANCA and IgA nephropathy in patients with crescentic GN [35]. Such patients more often have systemic signs and tend to have worse kidney function at presentation as compared to ANCA negative IgA nephropathy patients $[3,5]$. However, they tend to show better response to immunosuppressive medication $[5,6]$. From a prognostic and therapeutic standpoint, it is thus important that an attempt is made to determine the presence of the dominant pathology in such patients of crescentic GN.

Gastrointestinal involvement is rare in IgA vasculitis (unless associated with Henoch Schoenlein purpura [7,8]). It is also not common in ANCA vasculitis with anti MPO antibodies [7].

*Corresponding author: Mayank Chawla, Associate Consultant, Renal Medicine, Department of General Medicine, Sengkang General Hospital, 110 Sengkang East Way, Sengkang, 544886, Singapore

Accepted: October 06, 2020

Published online: October 08, 2020

Citation: Chawla M, Hoon TP, Baikunje S (2020) A Patient of Crescentic Glomerulonephritis with Positive Anti Myeloperoxidase Antibodies and Immunoglobulin a Deposits on Immunofluorescence: The Conundrum Continues. Ann Nephrol 5(1):71-74 
Citation: Chawla M, Hoon TP, Baikunje S (2020) A Patient of Crescentic Glomerulonephritis with Positive Anti Myeloperoxidase Antibodies and Immunoglobulin a Deposits on Immunofluorescence: The Conundrum Continues. Ann Nephrol 5(1):71-74

Table 1: Laboratory parameters.

\begin{tabular}{|l|l|l|}
\hline Parameter & Result & Normal value \\
\hline Hemoglobin (grams/deciliter) & 8.6 & $12-16$ \\
\hline Serum urea (milimol/liter) & 14.6 & $2.7-6.9$ \\
\hline Serum creatinine (micromol/liter) & 315 & $44-80$ \\
\hline Urine red blood cells (per microliter) & 198 & $0-4$ \\
\hline 24 hour total urine protein (grams per day) & 2.54 & $<0.13$ \\
\hline ELISA ${ }^{*}$ anti MPO (Ru/milliliter) & 169.5 & $<20$ \\
\hline ELISA $^{*}$ anti proteinase -3 (Ru/milliliter) & 5.4 & $<20$ \\
\hline
\end{tabular}

*Enzyme linked immunosorbent assay (performed by Euro immune analyzer).

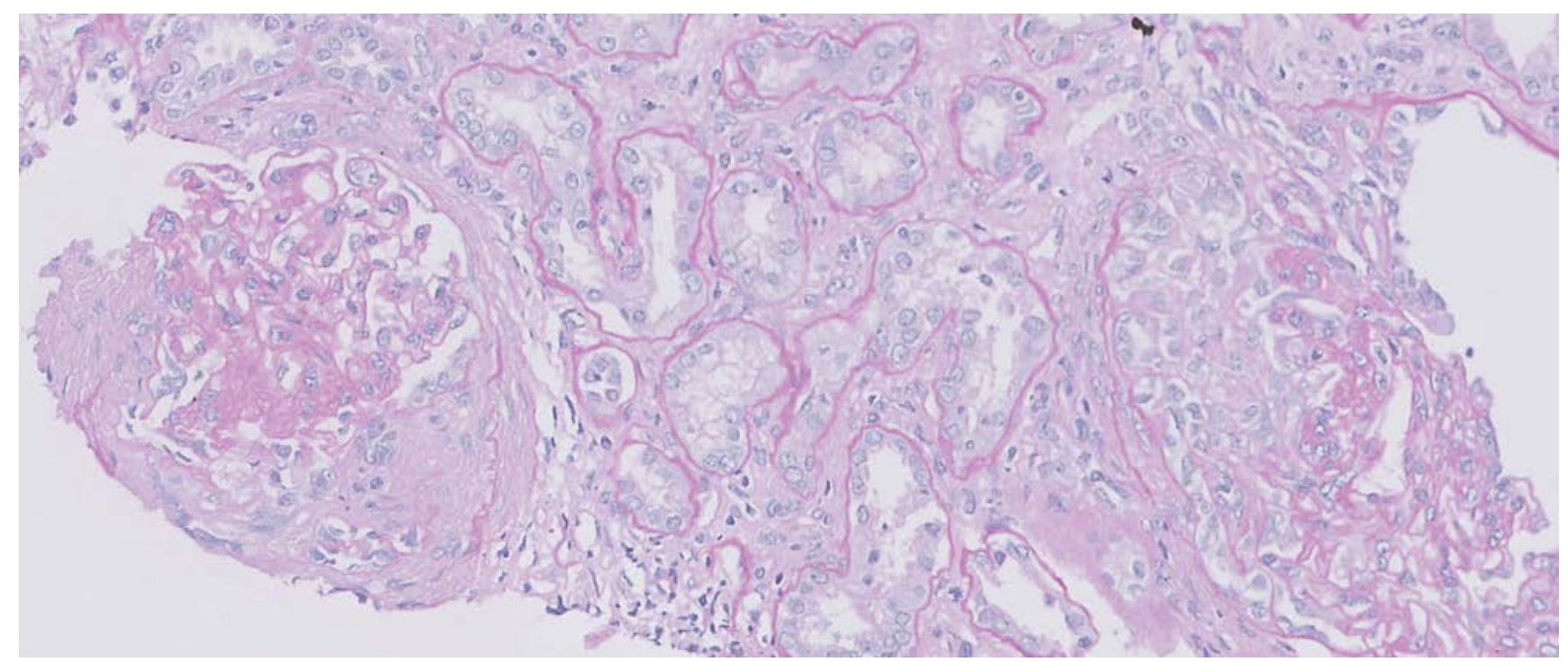

Figure 1: Renal biopsy, light microscopy (LM); Periodic acid-Schiff (PAS) stain: Two glomeruli, both with segmental sclerosis, with the glomerulus in the left field displaying a fibrocellular crescent and the glomerulus in the right field demonstrating a cellular crescent.

We report such a patient who presented with crescentic GN with IgA deposits on the IF and MPO serological positivity along with terminal ileitis.

\section{Case Presentation}

A 31-year-old Asian lady presented to us with crampy abdominal pain and non-bloody diarrhea for 1 month. She denied any fever, night sweats or loss of weight. There was no history of small joint pain, rash, and no stigmata of autoimmune disease. She was normotensive on admission and her systemic examination was unremarkable. ble 1.

Her significant laboratory parameters are indicated in Ta-

Her last known serum creatinine was 95 micromol per liter a few months ago. Her serum complement levels were normal. She was noted to be immune to Hepatitis B and had negative Hepatitis $C$ and HIV serologies.

A left kidney biopsy was performed using a Bard- Max -Core automated system with a 16-gauge needle.

The core sent for light microscopy (LM) contained 12 glomeruli out of which 4 were globally sclerotic. 4 glomeruli showed crescents ( 3 cellular and 1 fibrocellular) and 2 other

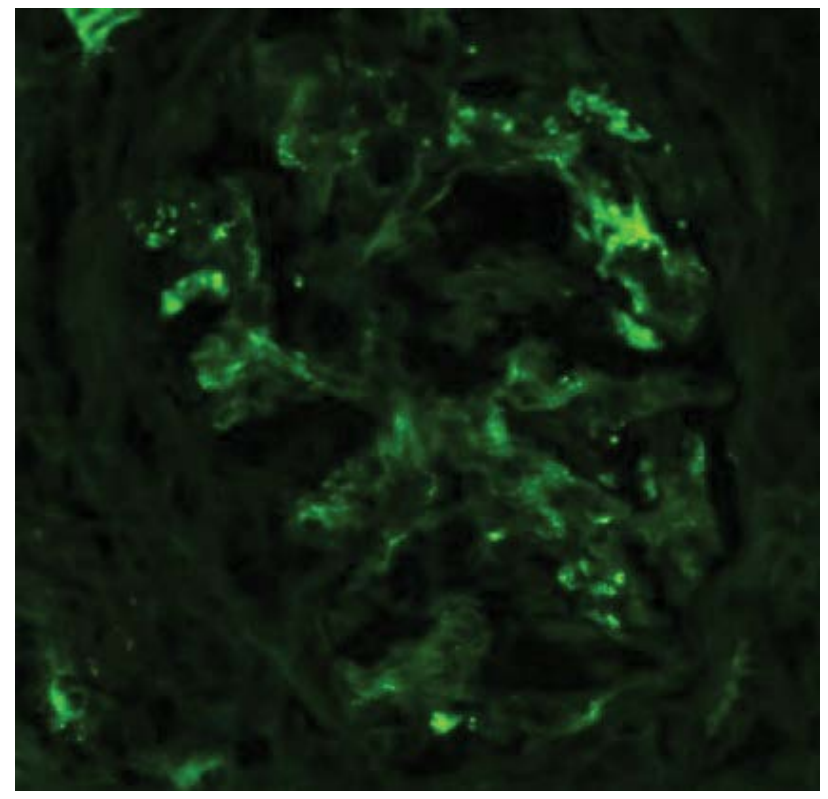

Figure 2: Renal Biopsy, IF for IgA showing mesangial staining 2+ - 3+ intensity. 
Citation: Chawla M, Hoon TP, Baikunje S (2020) A Patient of Crescentic Glomerulonephritis with Positive Anti Myeloperoxidase Antibodies and Immunoglobulin a Deposits on Immunofluorescence: The Conundrum Continues. Ann Nephrol 5(1):71-74

glomeruli showed features of early crescent formation. 5 out of the 12 glomeruli showed mild mesangial hypercellularity. The IF study revealed positive mesangial staining for IgA $(2-3+)$, IgM (2-3+) and C3 (2-3+) without any significant glomerular capillary wall staining. Ultrastructural study revealed deposits in the mesangial and paramesangial areas (Figure 1 and Figure 2).

She underwent a gastrointestinal (GI) tract evaluation for anemia. Her gastroscopy was normal. The colonoscopy revealed ulcers in the terminal ileum with a short segment of erythema. Histopathological analysis of these lesions was suggestive of a moderate mixed inflammatory cell infiltrate within the lamina propria, without any granuloma, giantcells, ischemia, or cytoplasmic inclusions.

The patient was initiated on pulse intravenous methylprednisolone $500 \mathrm{mg}$ once daily for 3 days followed by oral prednisolone at a dose of $60 \mathrm{mg}$ once daily for a period of 4 weeks after which the dose was tapered at regular intervals.

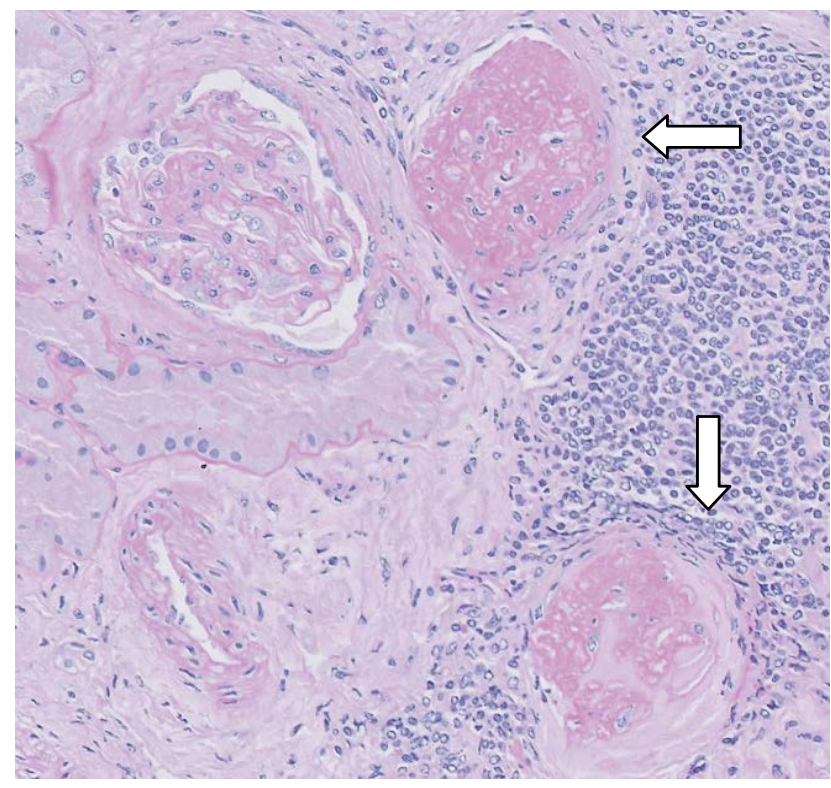

Figure 3: Renal biopsy, LM; PAS stain: Glomerular segmental sclerosis and two globally sclerotic glomeruli (arrows). There are chronic inflammatory cells within the fibrous interstitium.
It was decided to treat her with 6 doses of intravenous cyclophosphamide at a dose of $500 \mathrm{mg}$ per $\mathrm{m}^{2}$ of body surface area, at monthly intervals. She received a cumulative dose of 6.25 grams. She was maintained on ace inhibition throughout this period.

Her abdominal pain and diarrhea resolved after a few days of steroid therapy. Her anti MPO titer decreased to $32.4 \mathrm{Ru} /$ $\mathrm{ml}$ after 4 months of treatment but her renal function did not show any improvement after 6 months and hence we decided to repeat her renal biopsy (Graph 1).

Her repeat biopsy showed marked chronic changes in her glomeruli without crescents. There was moderate to marked tubular atrophy and interestingly there was no mesangial IgA staining on IF (Figure 3 and Figure 4).

\section{Discussion}

This patient demonstrated an interesting combination of renal and GI tract pathologies. She had crescentic glomerulonephritis as demonstrated by $50 \%$ crescents on her renal

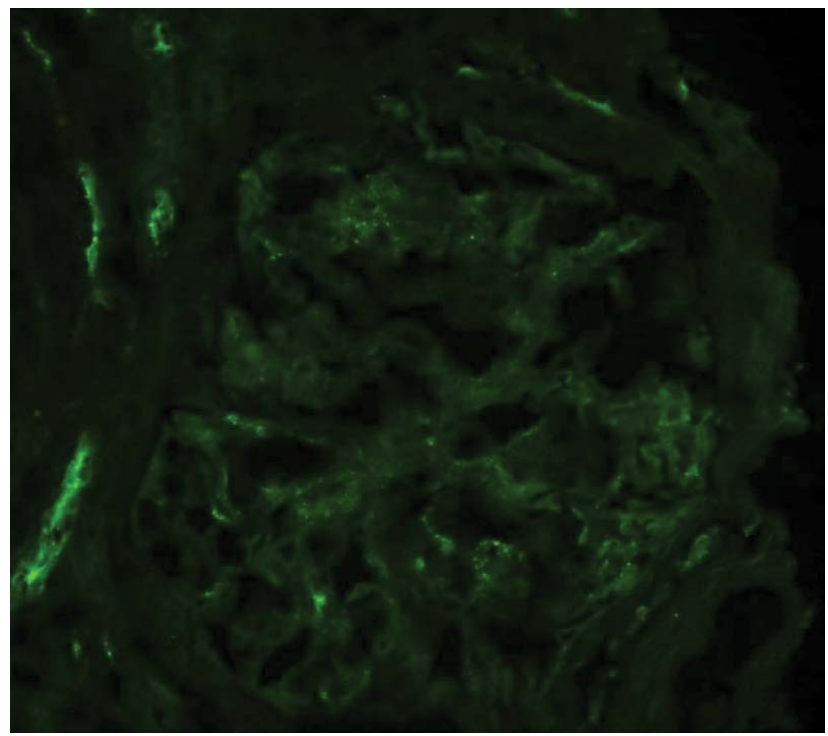

Figure 4: Renal biopsy, IF for IgA showing weak glomerular segmental mesangial reactivity $(1+)$.

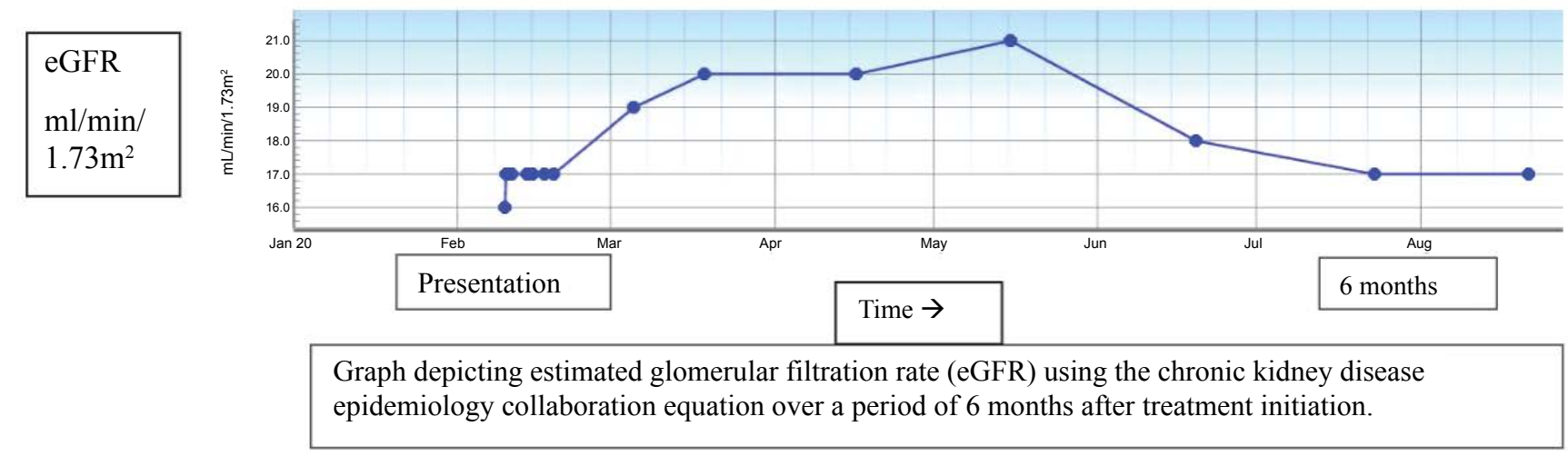

Graph 1: Graph depicting estimated glomerular filtration rate (eGFR) using the chronic kidney disease epidemiology collaboration equation over a period of 6 months after treatment initiation. 
Citation: Chawla M, Hoon TP, Baikunje S (2020) A Patient of Crescentic Glomerulonephritis with Positive Anti Myeloperoxidase Antibodies and Immunoglobulin a Deposits on Immunofluorescence: The Conundrum Continues. Ann Nephrol 5(1):71-74

biopsy. There were features of IgA nephropathy based on dominant IgA staining on IF and presence of mesangial and paramesangial deposits on the ultrastructural study along with positive serum anti MPO antibodies. She was also diagnosed with terminal ileitis. The diagnostic challenge was to decide whether the presence of MPO antibodies was purely coincidental in a patient with crescentic IgA nephropathy or whether this was a case of ANCA- associated crescentic GN with incidental IgA deposits in the mesangium.

Terminal ileal involvement can be seen in Henoch Schoenlein purpura [7], however our patient did not have the typical purpuric rash, which is an essential diagnostic criterion. Although the ileal biopsy was able to rule out Tuberculosis, it did not contribute further information since IF was not performed.

Our patient had severe renal dysfunction due to crescentic $\mathrm{GN}$, hence we chose to treat her with cyclophosphamide even though she fell in the reproductive age group. This was the best option available to us for treatment of crescentic GN at that time based on the international guidelines [9]. However, recent evidence has shown that rituximab is an equally effective option in patients with severe renal dysfunction [10].

Haas and colleagues previously reported 6 similar cases of crescentic nephritis with positive ANCA and IgA deposits, out of which 2 patients had MPO antibodies. They compared the renal biopsy findings with patients of crescentic IgA nephropathy without ANCA and found that the patients with ANCA positivity had lesser degree of endocapillary proliferation and mesangial hypercellularity whereas there was no difference in the number of glomeruli with crescents [6]. Our patient also had mild mesangial hypercellularity and endocapillary proliferation. The IgA staining was not very intense in the first biopsy, becoming even less so in the second biopsy. Bantis and colleagues have reported no difference in the intensity of mesangial IgA deposits between MPO positive and negative patients with crescentic IgA nephropathy [5]. While the reduced staining in the second biopsy of our patient may be related to treatment, it could also suggest that IgA was not a dominant pathology.

The renal function of our patient did not improve after induction therapy with cyclophosphamide, which is different from what was observed by Bantis and colleagues [5], who compared 8 patients with crescentic GN with mesangial IgA deposits associated with anti MPO antibodies with 26 patients with crescentic IgA nephropathy without anti MPO antibodies. The renal function of all the MPO positive crescentic IgA nephropathy patients improved after 6 months.
In conclusion, it is indeed challenging to interpret this combination of positive anti MPO antibodies with mesangial hypercellularity, positive mesangial staining for IgA and presence of crescentic nephritis. It is possible that this patient developed ANCA associated crescentic GN on a background of IgA nephropathy, since IgA nephropathy is common worldwide, and many such patients do not present with any renal dysfunction. The conundrum faced by the clinician in managing patients with coexisting IgA and ANCA related nephropathy continues.

\section{Acknowledgements}

Nil.

\section{References}

1. Anita Mc Grogan, Casper FM Franssen, Corinne S De Vries (2011) The incidence of primary glomerulonephritis worldwide: A systematic review of the literature. Nephrol Dial Transplant 26: 414-430.

2. Wang Feng, Xiang Tao, Li Junhui, et al. (2014) Coexisting glomerular IgA deposition and Ig G-kappa multiple myeloma. Ren Fail 36: 1345-1347.

3. Lijiao Xie, Jianghua He, Xing Liu, et al. (2018) Clinical value of systemic symptoms in IgA nephropathy with ANCA positivity. Clin Rheumatol 37: 1953-1961.

4. Ramirez SB, Rosen S, Niles J, et al. (1998) IgG antineutrophil cytoplasmic antibodies in IgA nephropathy: A clinical variant. Am J Kidney Dis 31: 341-344.

5. Christos Bantis, Maria Stangou, Christoph Schlaugat, et al. (2010) Is presence of ANCA in crescentic IgA nephropathy a coincidence or novel clinical entity? A case series. Am J Kidney Dis 55: 259268.

6. Mark Haas, Jabeen Jafri, Sharon M Bantosh, et al. (2000) ANCA-associated crescentic glomerulonephritis with mesangial IgA deposits. Am J Kidney Dis 36: 709-718.

7. Steven DiLauro, Nancy F Crum-Cianflone (2010) lleitis: When it is not Crohn's disease. Current Gastroenterol Rep 12: 249-258.

8. John P Thomas, Zay Htet (2020) Immunoglobulin A vasculitis presenting as terminal ileitis in late adulthood. J R Coll Physicians Edinb 50: 156-168.

9. (2012) Kidney disease improving global outcomes (KDIGO) guideline on glomerulonephritis. Kidney international.

10. Marta Casal Moura, Maria V Irazabal, Alfonso Erin, et al. (2020) Efficacy of Rituximab and plasma exchange in ANCA associated vasculitis with severe renal disease. J Am Soc Nephrol.

DOI: $10.36959 / 832 / 402$

Copyright: (C) 2020 Chawla M, et al. This is an open-access article distributed under the terms of the Creative Commons Attribution License, which permits unrestricted use, distribution, and reproduction in any medium, provided the original author and source are credited. 